\title{
Multiple Circular Colouring as a Model for Scheduling
}

\author{
Bing Zhou \\ Department of Mathematics, Trent University, Peterborough, Canada \\ Email: bzhou@trentu.ca
}

Received April 30, 2013; revised May 31, 2013; accepted June 15, 2013

Copyright (C) 2013 Bing Zhou. This is an open access article distributed under the Creative Commons Attribution License, which permits unrestricted use, distribution, and reproduction in any medium, provided the original work is properly cited.

\begin{abstract}
In this article we propose a new model for scheduling periodic tasks. The model is based on a variation of the circular chromatic number, called the multiple circular colouring of the conflict graph. We show that for a large class of graphs, this new model will provide better solutions than the original circular chromatic number. At the same time, it allows us to avoid the difficulty of implementation when the fractional chromatic number is used.
\end{abstract}

Keywords: Graph Coloring; Circular Chromatic Number; Fractional Chromatic Number; Multi-Circular Coloring; Scheduling Problem

\section{Introduction}

We consider the scheduling problems involving tasks $T_{1}, T_{2}, \cdots, T_{n}$. If two tasks both use a common resource, they cannot be scheduled at the same time. A valid scheduling is a mapping $f$ from $\left\{T_{1}, T_{2}, \cdots, T_{n}\right\}$ to the subsets of a time period $[0, \mathrm{~T}]$ such that

$f\left(T_{i}\right) \cap f\left(T_{j}\right)=\varnothing$ if $T_{i}$ and $T_{j}$ use a common resource. Let the value of a scheduling $f$ be

$|f|=\min \left\{\left|f\left(T_{i}\right)\right| / T: 1 \leq i \leq n\right\}$, which is the minimum

length of time a task has been assigned normalized by the length of the time period. The goal is to find a scheduling $f^{*}$ that maximizes $|f|$. One example of this type of scheduling problem is the heavily loaded resource sharing system in computer science [1-3]. The tasks are processes and some of them may share a common data file. Two processes that do share a common data file cannot operate at the same time. A scheduling of the processes that has the maximum value would allow the processes to operate most efficiently.

The constraints of the scheduling problem can be represented by a graph, called the conflict graph. The conflict graph $G$ has vertex set $\left\{v_{1}, v_{2}, \cdots, v_{n}\right\}$ representing the tasks where two vertices $v_{i}$ and $v_{j}$ are adjacent if and only if they use at least one common resource. Vertex colouring and chromatic numbers of the conflict graph have been used as models for scheduling problems (see for example [4]). If $G$ can be coloured with $k$ colours such that no two adjacent vertices have the same colour, we can then divide $[0, T]$ into $k$ equal length periods. All vertices that are coloured with the same colour do not have edges between them and therefore can be assigned to one period. For this scheduling $f$, we have $|f|=1 / \chi(G)$. Thus in general $\left|f^{*}\right| \geq 1 / \chi(G)$.

When the tasks are periodic in nature, circular colouring of the conflict graph is a more appropriate method. Circular colouring and the circular chromatic number (also called the star chromatic number) were introduced by A. Vince in 1988 [5]. A $(k, d)$-circular-colouring of a graph $G$ is a mapping $c: V(G) \rightarrow\{0,1, \cdots, k-1\}$ such that for each edge $x y$ in $G, d \leq|c(x)-c(y)| \leq k-d$. The circular chromatic number of $G, \chi_{c}(G)$, is the infimum of the ratio $k / d$ for which $G$ has a $(k, d)$-circularcolouring.

Equivalently, we can consider a $(k, d)$-circular-colouring of $G$ as a mapping $c$ from $V(G)$ to the open arcs of length $d$ in a circle of length $k$ such that if $x y$ is an edge in $G$, $c(x) \cap c(y)=\varnothing$. If we assign the tasks using a $(k, d)$ circular-colouring of the conflict graph $G$, we would have $|f| \geq d / k=1 / \chi_{c}(G)$.

A $(k, d)$-set-colouring of a graph $\mathrm{G}$ is a mapping $c$ such that for every vertex $v$ in $G, c(v)$ is a $d$-subset of

$\{0,1, \cdots, k-1\}$ and if $x y$ is an edge in $G, c(x) \cap c(y)=\varnothing$. The fractional chromatic number of a graph $G, \chi_{f}(G)$, is the infimum of the ratio $(k / d)$ for which $G$ has a $(k, d)$-set-colouring. If we can consider a $(k, d)$-set-colouring as a mapping $c$ from $V(G)$ to the sets of arcs in the circle of length $k$ such that the length of the union of arcs in $f(v)$ is at least $d$ for every vertex $v$ and if $x y$ is an edge in $G, c(x) \cap c(y)=\varnothing$. An assignment using a $(k, d)$-set-colouring of the conflict graph $G$ would yield 
$|f| \geq d / k=1 / \chi_{f}(G)$.

Circular chromatic number and fractional chromatic number and their variations have been extensively studied in the last two decades [6-11]. More results on the circular chromatic number and fractional chromatic number can be found in the book [12] and the survey papers $[13,14]$. It is easy to see that by their definition, we have the relations

$$
\chi_{f}(G) \leq \chi_{c}(G) \leq \chi(G) .
$$

Vince proved in [5] that

$$
\chi(G)-1<\chi_{c}(G) \text {. }
$$

Therefore, we have

$$
\chi(G)=\chi_{c}(G) .
$$

While the difference between $\chi(G)$ and $\chi_{c}(G)$ is always less than 1 , it is known that the difference between $\chi(G)$ and $\chi_{f}(G)$ can be arbitrarily large for some graphs. An example is the family of graphs called Kneser graphs. A Kneser graph $([15,16]) K_{p, q}$ has all $q$-subsets of $\{0,1, \cdots, p-1\} \quad(p>2 q)$ as its vertices and two vertices are adjacent if the two subsets are disjoint. It is proved in [16] that

$$
\begin{gathered}
\chi_{f}(K(p, q))=\frac{p}{q}, \\
\chi(K(p, q))=p-2 q+2 .
\end{gathered}
$$

Another example of a family of graphs where the difference between their chromatic number and fractional chromatic number is large is the graphs obtained by using Mycielski's construction.

For a graph $G$ with vertex set $V(G)=\left\{u_{1}, u_{2}, \cdots, u_{n}\right\}$ and edge set $E(G)$, the Mycielskian $M(G)$ of $G$ is the graph with vertex set

$\left\{u_{1}, u_{2}, \cdots, u_{n}\right\} \bigcup\left\{v_{1}, v_{2}, \cdots, v_{n}\right\} \bigcup\{w\}$ and edge set $E \bigcup\left\{u_{i}, v_{j}: u_{i}, u_{j} \in E\right\} \bigcup\left\{v_{i} w: i=1,2, \cdots, n\right\} . M\left(C_{5}\right)$ in

Figure 1 is also called the Grotzsch graph.

It is well known that

$$
\chi(M(G))=\chi(G)+1 .
$$

For the fractional chromatic number of Mycielskians, the authors of [17] found the recurrence relation

$$
\chi_{f}(M(G))=\chi_{f}(G)+1 / \chi_{f}(G) .
$$

For the Grotzsch graph, since $\chi_{f}\left(C_{5}\right)=5 / 2$, we have

$$
\chi_{f}\left(M\left(C_{5}\right)\right)=5 / 2+2 / 5=29 / 10 .
$$

We use the notation $M^{t}(G)$ such that

$$
M^{t}(G)=\underbrace{M(M(\cdots M}_{t \text { times }}(G))) .
$$

We have

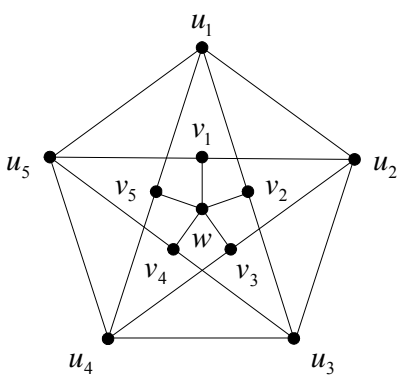

Figure 1. Grotzsch graph.

$$
\chi_{f}\left(M^{t}\left(C_{5}\right)\right) \leq 5 / 2+t / 2 .
$$

In comparison, we have

$$
\chi\left(M^{t}\left(C_{5}\right)\right)=3+t .
$$

For this class of graphs, the difference between $\chi$ and $\chi_{f}$ is unbounded.

For the circular chromatic number, we have

$$
\chi_{c}\left(M^{t}\left(C_{5}\right)\right)>\chi\left(M^{t}\left(C_{5}\right)\right)-1=2+t .
$$

This shows that the difference between the circular chromatic number and the fractional chromatic number is also arbitrarily large for the Mycielskians. To achieve the optimal result for a scheduling problem in general, it appears that the fractional chromatic number of the conflict graph provides the best results.

However, there are difficulties if we use $(k, d)$-set colouring and the fractional chromatic number in the scheduling problem. To achieve $\chi_{f}(G)$ the optimal $(k, d)$-set colouring may have a very large value of $d$. As pointed out in [12], $M^{t}\left(C_{5}\right)$ is an example of a graph $G$ for which $\chi_{f}(G)=k / d$ for no small $d$. In fact, if we let $G_{2}=C_{5}$ and $G_{n}=M\left(G_{n-1}\right)$, then

$$
\left|V\left(G_{n}\right)\right|=3 \cdot 2^{n-1}-1
$$

and $\chi_{f}\left(G_{n}\right)$ is a fraction whose denominator, when written in smallest terms, is greater than $2^{2^{n-2}}$. This example shows that there is no bound on the denominator of $\chi_{f}(G)$ that is a polynomial function of the number of vertices of $G$. If this optimal set colouring is to be used for scheduling, each task would be divided into too many fragments making it impossible in practice. To combine optimality and practicality, in the next section we propose a new colouring of the conflict graph that will provide a better solution than the circular colouring and easier to implement than the set colouring.

\section{Multiple Circular Colouring of a Graph}

Definition 1 An $m$ - $(k, d)$-circular colouring of a graph $G$ is a mapping c from $V(G)$ to the sets of open arcs in the circle of length $k$ such that $c(v)$ is the union of $m$ arcs with a total length at least $d$ and if $x y$ is an edge in $G$, 
$c(x) \cap c(y)=\varnothing$. The m-circular chromatic number, $\chi_{c}^{m}(G)$ is the infimum of the ration $k / d$ for which Ghas a $m$ - $(k, d)$-circular-colouring.

It is easy to see that for every positive integer $m$,

$$
\chi_{f}(G) \leq \chi_{c}^{m}(G) \leq \chi_{c}(G) .
$$

To demonstrate this colouring indeed improves the solution of the scheduling problem in some cases, we show that even for $m=2, \chi_{c}^{2}(G)$ can be strictly less than $\chi_{c}(G)$ for large classes of graphs.

Recall that for a graph $G, M(G)$ is the Mycielskian of $G$. There are many graphs $G$ such that $\chi_{c}(M(G))=\chi(M(G))$. Some sufficient conditions for this equality to hold are given in, for example, [18] and [19]. However, $\chi_{c}^{2}(M(G))$ will always be strictly less than $\chi(M(G))$.

Theorem 2 For every graph $G$,

$$
\chi_{c}^{2}(M(G)) \leq \chi(M(G))-\frac{1}{2} .
$$

Proof: Let $V(G)=\left\{u_{1}, u_{2}, \cdots, u_{n}\right\}, \chi(G)=k$ and $V(M(G))=\left\{u_{1}, u_{2}, \cdots, u_{n}\right\} \bigcup\left\{v_{1}, v_{2}, \cdots, v_{n}\right\} \bigcup\{w\}$ as described in the previous section. Let $[0, k)$ be a circle of length $k$. Since $\chi(G)=k$, there is a mapping of the form $c\left(u_{i}\right)=(j, j+1)$ for each $i$ where $j \in\{0,1, \cdots, k-1\}$ such that $c\left(u_{i_{1}}\right) \cap c\left(u_{i_{2}}\right)=\varnothing$ whenever $u_{i_{1}}$ and $u_{i_{2}}$ are adjacent.

Let $\left[0, k+\frac{1}{2}\right)^{2}$ be a circle of length $k+1 / 2$. Let

$c(w)=\left(0, \frac{1}{2}\right) \cup\left(1, \frac{3}{2}\right)$. Notice that $\left|c(w) \cap c\left(u_{i}\right)\right| \leq \frac{1}{2}$

for all $i$. Figure 2 demonstrates this mapping when $G=C_{5}$.

For each $i$, if $c\left(u_{i}\right) \cap c(w)=\varnothing$, let $c\left(v_{i}\right)=c\left(u_{i}\right)$; otherwise, let $c\left(v_{i}\right)=\left\{c\left(u_{i}\right) \backslash c(w)\right\} \cup(k, k+1 / 2)$. We show that the arcs representing these vertices in the case of $G=C_{5}$ in Figure 3 .

It is easy to check that this is a valid 2-( $\left.\frac{2 k+1}{2}\right)$-circular colouring of $M(G)$ in general. This proves that

$$
\chi_{c}^{2}(M(G)) \leq k+\frac{1}{2}=\chi(M(G))-\frac{1}{2} .
$$

Corollary 3 If $\chi_{c}(M(G))=\chi(M(G))$, then

$$
\chi_{c}^{2}(M(G)) \leq \chi_{c}(M(G))-\frac{1}{2} .
$$

Next we show that unlike the set-chromatic number, the denominator of the $m$-circular chromatic number is bounded by the product of $\mathrm{m}$ and the number of vertices in the graph.

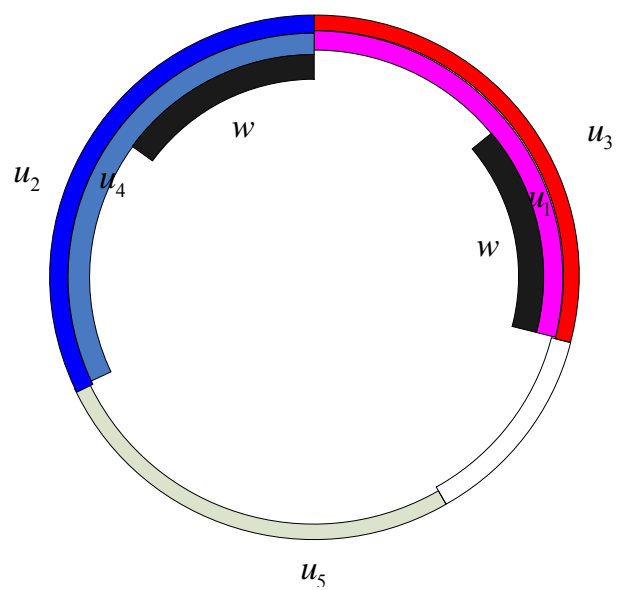

Figure 2. A 2-circhular coloring of vertices of $C_{5}$ and $w$ in $M$ $\left(C_{5}\right)$.

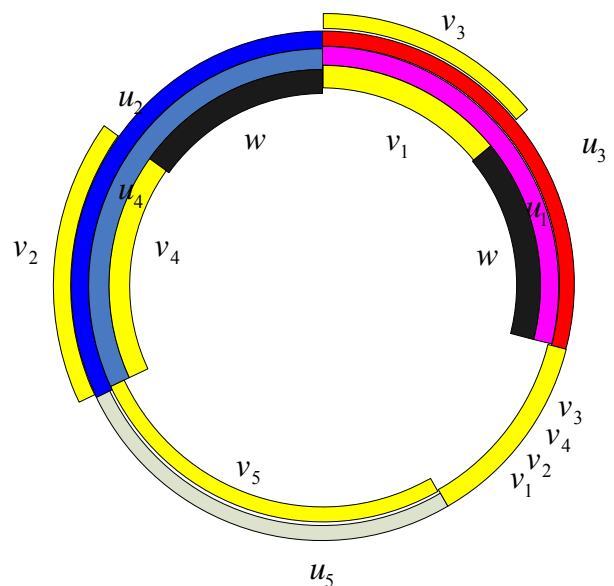

Figure 3. A 2-circular coloring of $M\left(C_{5}\right)$.

Theorem 4 Let $G$ be a graph of $n$ vertices, and $\chi_{c}^{m}(G)=\frac{k}{d}$. Then $k$ and $d$ can be integers such that $d<$ mn.

Proof: Suppose that $\chi_{c}^{m}(G)=r$. By scaling if necessary, there is a $m$-circular colouring $\mathrm{c}$ that maps the vertices of $G$ to sets of $m$ arcs in a circle of length $r$ such that each arc has length at least $\frac{1}{m}$ and if $x y$ is an edge then $c(x) \cap c(y)=\varnothing$. We assume that $c$ is a colouring such that the set

$$
\left\{l: l \text { is an arc in } c(v) \text { for some vertex } v \text { and }|l|=\frac{1}{m}\right\}
$$

is the smallest. We fix a direction of the circle, say counter clockwise.

There is at least one arc $l$ such that $|l|=\frac{1}{m}$; otherwise $r$ could be made smaller. Let that arc be $l_{1}$. There must be an arc $l_{2}$ such that i) $c^{-1}\left(l_{1}\right)$ is adjacent to $c^{-1}\left(l_{2}\right)$ in 
$G$, ii) the left end of $l_{1}$ is the right end of $l_{2}$ and iii) $\left|l_{2}\right|=\frac{1}{m}$, otherwise $\left|l_{1}\right|$ could be made larger.

Continuing this process, some arc will have to be used more than once. Say the first time this happens is at $l_{a}=l_{b}$. Then the arcs $l_{a}, l_{a+1}, \cdots, l_{b-1}$ must cover the circle an integer number of times. Suppose that they cover the circle $s$ times. Since there are $b-a$ arcs and they all have length $\frac{1}{m}$, we have

$$
s r=\frac{(b-a)}{m}
$$

and

$$
r=\frac{(b-a)}{s m}
$$

Since $s<n$, the denominator is less than $m n$.

Intuitively, when the value of $m$ increases, the $m$-circular chromatic number will be closer to the fractional chromatic number and thus the difference between the chromatic number and $m$-circular chromatic number would increase. Nevertheless, our next theorem shows that the $m$-circular chromatic number cannot be less than one $m$-th of the .chromatic number.

Theorem 5 For every graph $G, \quad \chi_{c}^{m}(G) \geq \frac{1}{m} \chi(G)$.

Proof: Suppose that $\chi_{c}^{m}(G)=r$. There is an $m$-circular colouringc such that $|c(v)| \geq \frac{1}{r}$ for every vertex $v$. Since $c(v)$ is a union of $m$ arcs, at least one of the arcs has length at least $\frac{1}{m r}$. We insert $m r$ points $P_{1}, P_{2}, \cdots, P_{m r}$ spaced at equal distance in the unit length circle. For every vertex $v, c(v)$ contains at least one of these points. Let $V_{i}=\left\{v \in G: c(v) \ni P_{i}\right\}$. Each $V_{i}$ is an independent set and $V(G)=U V_{i}$. G can be coloured with mrcolours. So we have

$$
\begin{gathered}
\chi(G) \leq m r=m \chi_{c}^{m}(G), \\
\text { i.e., } \chi_{c}^{m}(G) \geq \frac{1}{m} \chi(G) .
\end{gathered}
$$

The Kneser graphs provide examples showing this lower bound is asymptotically the best possible.

Let $\alpha(G)$ be the independence number of $G$. $\chi_{c}^{m}(G)$ is also bounded by a function of $\alpha(G)$. The proof above yields a lower bound for $\alpha(G)$.

Theorem 6 For every graph $G$ with $n$ vertices,

$$
\chi_{c}^{m}(G) \geq \frac{n}{m \alpha(G)} \text {. }
$$

Proof: The set $V_{i}$ in the proof of Theorem 5 is an in- dependent set. We have $m \chi_{c}^{m}(G)$ such independent sets. The average size of one set is $\frac{n}{m \chi_{c}^{m}(G)}$. Therefore

$$
\alpha(G) \geq \frac{n}{m \chi_{c}^{m}(G)} .
$$

and

$$
\chi_{c}^{m}(G) \geq \frac{n}{m \alpha(G)} .
$$

\section{Conclusion}

We proved that for a large class of graphs, this multiple circular colouring and $m$-circular chromatic number of the conflict graph will provide better solutions for the scheduling problem than the original circular chromatic number. It is also easier to implement than the model using the fractional chromatic number. We plan to investigate more classes of graph $G$ where $\chi_{c}^{m}(G)<\chi_{c}(G)$ for small values of $m$. It would also be interesting to find out the probability for random graphs to have $m$-circular chromatic number strictly less than their circular chromatic number.

\section{REFERENCES}

[1] V. C. Barbosa and E. Gafni, "Concurrency in Heavily Loaded Neighborhood-Constrained Systems," ACM Transactions on Programming Languages and Systems, Vol. 11, No. 4, 1989, pp. 562-584. doi:10.1145/69558.69560

[2] H. G. Yeh, "A Connection between Circular Colorin and Periodic Schedules," Discrete Applied Mathematics, Vol. 157, No. 7, 2009, pp. 1663-1668. doi:10.1016/i.dam.2008.10.003

[3] H. G. Yeh and X. Zhu, "Resource-Sharing System Scheduling and Circular Chromatic Number," Theoretical Computer Science, Vol. 332, No. 1-3, 2005, pp. 447-460. doi:10.1016/j.tcs.2004.12.005

[4] F. Chung, "Graph Theory in the Information Age," Notices of AMS, Vol. 57, No. 6, 2010, pp. 726-732.

[5] A. Vince, "Star Chromatic Number," Journal of Graph Theory, Vol. 12, No. 4, 1988, pp. 551-559. doi:10.1002/jgt.3190120411

[6] H. L. Abbott and B. Zhou, "The Star Chromatic Number of a Graph," Journal of Graph Theory, Vol. 17, No. 3, 1993, pp. 349-360. doi:10.1002/jgt.3190170309

[7] H. Hatami and R. Tusserkani, "On the Complexity of the Circular Chromatic Number," Journal of Graph Theory, Vol. 47, No. 3, 2004, pp. 226-230. doi:10.1002/jgt.20022

[8] K. Kilakos and B. Reed, "Fractionally Colouring Total Graphs," Combinatorica, Vol. 13, No. 4, 1993, pp. 435440. doi:10.1007/BF01303515

[9] D. Kral, E. Macajova and J.-S. Sereni, "Circular Edge- 
Coloring of Cubic Graphs with Girth Six," Journal of Combinatorial Theory, Series B, Vol. 100, No. 4, 2010, pp. 351-358. doi:10.1016/j.jctb.2009.10.003

[10] I. Leader, "The Fractional Chromatic Number of Infinite Graphs," Journal of Graph Theory, Vol. 20, No. 4, 1995 , pp. 411-418. doi:10.1002/jgt.3190200404

[11] B. Zhou, "Some Theorems Concerning the Star Chromatic Number of a Graph," Journal of Combinatorial Theory, Series B, Vol. 70, No. 2, 1997, pp. 245-258. doi:10.1006/jctb.1996.1738

[12] E. R. Scheinerman and D. H. Ullman, "Frational Graph Theory," John Wiley and Sons, New York, 1997.

[13] X. Zhu, "Circular Chromatic Number-A Survey," Discrete Mathematics, Vol. 229, No. 1-3, 2001, pp. 371-410. doi:10.1016/S0012-365X(00)00217-X

[14] X. Zhu, "Recent Developments in Circular Colouring of Graphs," Algorithms and Combinatorics, Vol. 26, Springer, Berlin, Heidelberg, 2006, pp. 497-550.
[15] M. Knser, "Aufgabe 300," Jahresbericht der Deutsche Mathematiker-Vereinigung, Vol. 58, No. 2, 1955, p. 27.

[16] L. Lovasz, "Kneser's Conjecture, Chromatic Number, and Homotopy," Journal of Combinatorial Theory, Series A, Vol. 25, No. 3, 1978, pp. 319-324. doi:10.1016/0097-3165(78)90022-5

[17] M. Lasen, J. Propp and D. H. Ullman, "The Fractional Chromatic Number of Mycielski Graphs," Journal of Graph Theory, Vol. 19, No. 3, 1995, pp. 411-416. doi:10.1002/jgt.3190190313

[18] G. Chang, "Circular Chromatic Numbers of Mycielski's Graphs," Discrete Mathematics, Vol. 205, No. 1-3, 205, 1999, pp. 23-37. doi:10.1016/S0012-365X(99)00033-3

[19] H. Jajiaholhassan and X. Zhu, "Circular Chromatic Number and Mycielski Construction," Journal of Graph Theory, Vol. 44, No. 2, 2003, pp. 106-115. doi:10.1002/jgt. 10128 